\begin{tabular}{ccc}
\hline International Journal of Engineering \& Technology, 7 (3.7) (2018) 303-306 \\
International Journal of Engineering \& Technology \\
Website: www.sciencepubco.com/index.php/IJET \\
Research paper
\end{tabular}

\title{
Robust RAM Control for WSR on the Water Surface
}

\author{
Kyunghan Chun and Bonghwan Kim* \\ School of Electronic and Electrical Engineering, Daegu Catholic University, Gyeongbuk 38430, Korea \\ *Corresponding author E-mail: bhkim@cu.ac.kr
}

\begin{abstract}
This paper discusses a robust rotate and move (RAM) controller by considering a water striding robot (WSR) with two wheels. The proposed controller commands the WSR to rotate and move straight toward the desired target by considering the two wheel WSR characteristics. Sliding mode control (SMC) is one of the solutions in nonlinear controller design and it has fast response and robustness. SMC is applied to the WSR RAM control. However, the sliding mode has a problem called chattering because of using sign function in controller design and this will cause the system unstable in WSR control because the chattering make the WSR sink into water easily. As a solution, sign function is replaced by saturation function. The proposed controller is noble and track the target point easily and also has robustness. The stability of the proposed controller is proved by Lyapunov function and the simulation results show the fast response and robustness.
\end{abstract}

Keywords: Two wheel, Tracking, WSR, RAM, SMC

\section{Introduction}

Researchers have recently focused on the surface-tension-driven locomotion of water-walking arthropods such as water striders and fisher spiders [1-8]. Various kinds of miniature robots, which are able to move on water, have been developed. Hu et al. identified the mechanism of the momentum transfer that was responsible for water strider locomotion and proposed a mechanical water strider driven by elastic thread [2]. Gao and Jiang [3] showed that the legs of water striders are covered with thousands of tiny hairs that have fine nanoscale grooves. These hierarchical micro- and nanostrucutres create super-hydrophobic surfaces. Suhr et al. developed a water strider robot that was driven in one of its resonant modes using unimorph piezoelectric actuators [4]. Song et al. analyzed the statics of the supporting leg and developed a nontethered water strider robot using two miniature DC motors and a lithium-polymer battery $[6,8]$.

To make water strider robot (WSR) slide on the water surface, the robot's dynamics modeling and control algorithms are discussed [9] and simple rotate and move (RAM) control method can be applied because the buoyance of the WSR is well affected by the system dynamics variation and also the variation makes WSR unstable and sinking into the water. So, the nonlinear control algorithm is required to obtain fast response and other good performance.

By the way, siliding mode control (SMC) is one of the nonlinear control solutions and has fast response and robustness [9-16]. Its robustness comes from the sign function, which is included in the controller. But it also causes so-called a chattering problem which makes the system unstable. So, the saturation function is used conventionally instead of sign function and in WSR control, the saturation function is more effective in stability and simple to apply.
In this paper, a SMC is developed and applied to the WSR including hydrophobic supporting legs with microstructured surfaces by using micro electromechanical systems (MEMS) techniques. Furthermore water strider insects have attracted many researchers' attention with their power efficient and agile water surface locomotion. So the WSR with twelve microfabricated legs driven by a vibration motor is simulated and the target tracking is performed successfully. The stability is proved by the Lyapunov function and the MATLAB/SIMULINK simulation shows the good performance.

\section{Model and Ram Control}

Model

For implementing RAM control, the cylindrical coordinate system is better than the rectangular coordinate system [9], because physical values for RAM (rotate and move) can be directly measured in the cylindrical coordinate system.

In the cylindrical coordinate system, the dynamics is formulated as Eq. 1.

$\ddot{\theta}=\frac{2 W}{I} F$

$\ddot{r}=\frac{2 F}{m}$

and also expressed as Eq. 2.

$\ddot{\theta}=\frac{2 W}{I} F=K_{\theta} u$ 
$\ddot{r}=\frac{2}{m} F=K_{r} u$

where

$K_{\theta}=\frac{2 W}{I} \quad K_{r}=\frac{2}{m} \quad u=F$

\section{RAM Control}

RAM control makes the WSR rotate and move, so the cylindrical coordinate system model of Eq. 2 is more convenient to apply than the rectangular coordinate system model.

Rotation) The WSR rotation can be classified into two cases by the angle absolute magnitude. One is the rotation of angle under $\pi$ and the other is the rotation of angle over $\pi$.

- Under $\pi\left(\left|\theta_{e}\right|<\pi\right)$, the rotation force is applied just to be proportional to the angle error $\theta_{e}$.

- Over $\pi\left(\left|\theta_{e}\right|>\pi\right)$, the force is applied to the following angle error of Eq. 4 for fast rotating.

$$
\theta_{e}=-\operatorname{sqn}\left(\theta_{e}\right)\left[2 \pi-|\theta|+\left|\theta_{d}\right|\right]
$$

Movement) The WSR movement is simple action to go straight toward the target point because the direction is exactly toward the target point by the rotation. So, the controller performance is enough to go straight only.

\section{Sliding Mode Control}

For SMC, there is sliding mode and reaching mode. In reaching mode, the state variable goes to the sliding surface from the initial condition at first and then on the sliding surface, the state goes to the origin and this is so called sliding mode [16]. So, in the sliding mode, the sliding surface should have a negative slope for the state to converge to the origin and in the reaching mode, the derivative of the sliding function should have the negative value to move toward to the sliding surface.

As mentioned in the previous chapter, model and RAM Control, the controllers for rotation and movement can be designed independently. So, for RAM control, the rotation controller is proposed first and the movement control is explained later.

Rotation) For rotation control, the angel error is Eq. 5

$$
\theta_{e}=\theta_{d}-\theta
$$

where $\theta_{d}$ is the desired angle. And for sliding control, we define the angle sliding function as Eq. 6 with the positive $\lambda_{\theta}$.

$$
s_{\theta}=\dot{\theta}_{e}+\lambda_{\theta} \theta_{e}
$$

To make the state goes to the sliding surface, the angle sliding mode controller is proposed as Eq. 7.

$u_{\theta}=\frac{1}{K_{\theta}}\left[-\lambda_{\theta} \dot{\theta}_{e}+Q_{\theta} \operatorname{sgn}\left(s_{\theta}\right)\right]$

where $Q_{\theta}$ is positive.

Theorem 1: For the dynamics of Eq. 2, the proposed controller of Eq. 7 with the sliding function of Eq. 6 satisfies the sliding condition $\dot{V}_{\theta}<0$.

Proof)
Let the Lyapunov function $V_{\theta}=\frac{1}{2} s_{\theta}{ }^{2}$ and by differentiating

$\dot{V}_{\theta}=s_{\theta} \dot{s}_{\theta}$

and Eq. 9 can be acquired by differentiating the sliding function of Eq. 6

$$
\begin{aligned}
\dot{s}_{\theta} & =\ddot{e}_{\theta}+\lambda_{\theta} \dot{e}_{\theta} \\
& =-\ddot{\theta}-\lambda_{\theta} \dot{\theta}
\end{aligned}
$$

Eq. 9 with Eq. 2 and Eq. 7 becomes

$$
\begin{aligned}
\dot{s}_{\theta} & =\lambda_{\theta} \dot{\theta}-Q_{\theta} \operatorname{sgn}\left(s_{\theta}\right)-\lambda_{\theta} \dot{\theta} \\
& =-Q_{\theta} \operatorname{sgn}\left(s_{\theta}\right)
\end{aligned}
$$

Therefore, by using Eq. 10, Eq. 8 results in

$$
\begin{aligned}
\dot{V}_{\theta} & =s_{\theta} \times-Q_{\theta} \operatorname{sgn}\left(s_{\theta}\right) \\
& =-Q_{\theta} s_{\theta} \operatorname{sgn}\left(s_{\theta}\right) \\
& <0
\end{aligned}
$$

Q.E.D

Movement) For movement control, the distance error is Eq. 12

$r_{e}=r_{d}-r$

where $r_{d}$ is the desired distance and for movement sliding mode control, the sliding function is defined as Eq. 13.

$s_{r}=\dot{r}_{e}+\lambda_{r} r_{e}$

where $\lambda_{r}$ is positive and the movement control input is proposed as Eq. 14.

$u_{r}=\frac{1}{K_{r}}\left[-\lambda_{r} \dot{r}_{e}-Q_{r} \operatorname{sgn}\left(s_{r}\right)\right]$

Where $Q_{r}$ is positive.

Theorem 2: For the dynamics of Eq. 2, the proposed controller of Eq. 14 with the sliding function of Eq. 13 satisfies the sliding condition $\dot{V}_{r}<0$.

Proof)

Let the Lyapunov function $V_{r}=\frac{1}{2} s_{r}{ }^{2}$ and by differentiating

$\dot{V}_{r}=s_{r} \dot{s}_{r}$

and Eq. 16 results from differentiating the sliding function of Eq. 13.

$\dot{s}_{r}=\ddot{r}+\lambda_{r} \dot{r}$

Eq. 16 with Eq. 2 and of Eq. 14 becomes

$$
\begin{aligned}
\dot{s}_{r} & =-\lambda_{r} \dot{r}-Q_{r} \operatorname{sgn}\left(s_{r}\right)+\lambda_{r} \dot{r} \\
& =-Q_{r} \operatorname{sgn}\left(s_{r}\right)
\end{aligned}
$$

Therefore, by using Eq. 17, Eq. 15 results in 


$$
\begin{aligned}
\dot{V}_{r} & =s_{r} \times-Q_{r} \operatorname{sgn}\left(s_{r}\right) \\
& =-Q_{r} s_{r} \operatorname{sgn}\left(s_{r}\right) \\
& <0
\end{aligned}
$$

\section{Simulation}

For WSR simulation, the model is Eq. 2 and the dc motor is used in [16]. For the model and the control force, the used all parameter values are as following:

$$
\begin{aligned}
& m: 5.03 \mathrm{~g}, W: 0.025 \mathrm{~m}, m_{R}: 0.3 \mathrm{~g}, \mathrm{a}=0.25, h: 0.015 \mathrm{~m}, \\
& \theta_{u w}=\frac{\pi}{2} \mathrm{rad}, I=\frac{m W^{2}}{6}
\end{aligned}
$$

and the computed force is

$F=1.4921 \times 10^{-10} n^{2}$

where $n$ is motor speed [r/min].

For the simulation of the proposed RAM SMC, the WSR model and RAM SMC are implemented by using MATLAB/SIMULINK and the target point is assumed as $(-5,-5)$. The used SIMULINK block for RAM SMC is shown in Fig. 1 and Fig. 1(a) is rotation SMC, Fig. 1(b) is movement SMC. As shown in Fig. 1, the same structure is applied, but the used parameters are properly selected as follows

$\lambda_{\theta}=5, Q_{t h}=10, \lambda_{r}=1, Q_{r}=0.1$

and the saturation function is applied in the simulation with the boundary layer.

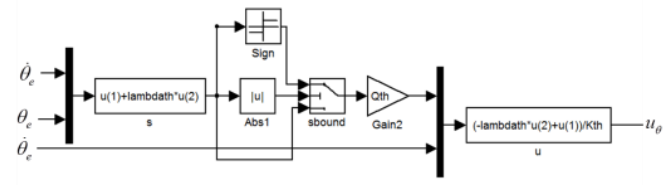

(a) Rotation SMC

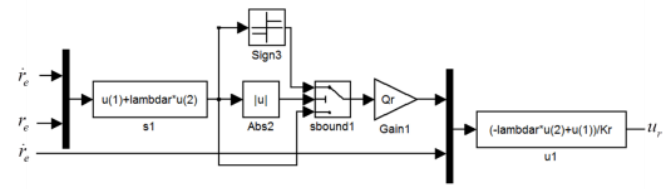

(b) Movement SMC

Fig. 1: Block diagram of RAM SMC in SIMULINK

For verifying the RAM steps in the control process, the angle and the $\mathrm{x}, \mathrm{y}$ positions are measured and displayed in the Fig. 2 to Fig. 4. In Fig. 2, at first, the angle tracking is started and after arriving target angle at about $2 \mathrm{~s}$, the angle SMC controller is switched OFF and the moving SMC controller makes the WSR go toward the target point in Fig. 3 and Fig. 4.

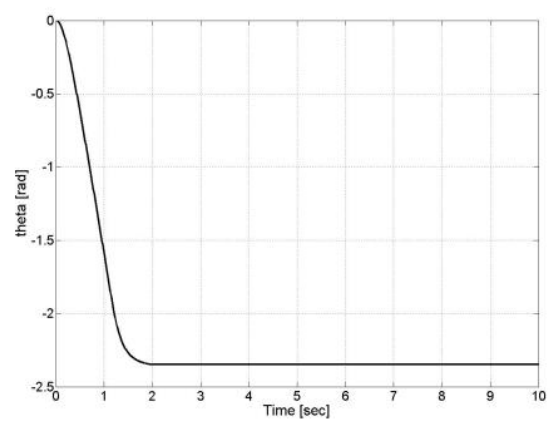

Fig. 2: Rotation

As shown in Fig. 2, the rotation is negative direction and the rotation value is $135^{\circ}$. This is the fast rotate direction in the given example. If the rotation is in the positive direction, it also can track the desired angle, but the response will be slower than this example because the rotation value will be $225^{\circ}$, which is larger than $135^{\circ}$

After finishing angle control, the moving control starts and two driving legs only works for the moving action because the WSR direction is now toward the target point. In Fig. 3 and Fig. 4, as we assumed that the target point $\mathrm{x}$ and $\mathrm{y}$ have the same values, the changing positions of $\mathrm{x}$ and $\mathrm{y}$ are almost same and after arriving target point, the moving control is also switched OFF at $5.3 \mathrm{~s}$.

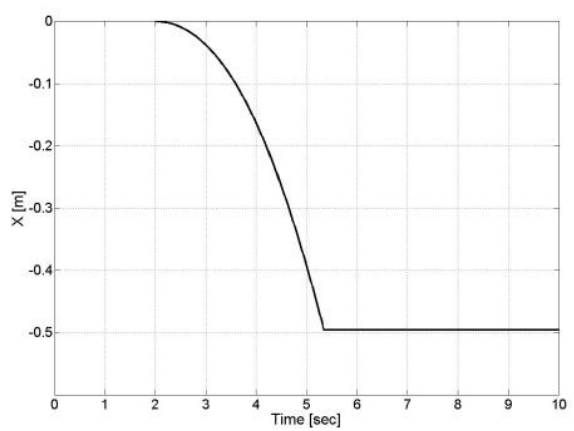

Fig. 3: Moving $-\mathrm{x}$ position

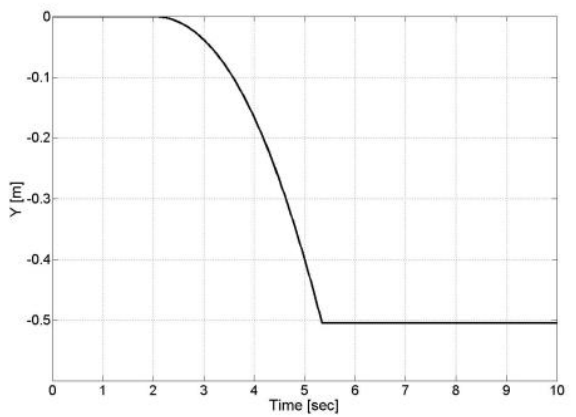

Fig. 4: Moving - y position

\section{Conclusion}

In this paper, we propose a robust method for controlling the movement of a WSR and considering the characteristics of the model. In the proposed method, RAM control is used in consideration of the characteristics of the two - wheel drive system [9]. And furthermore, for the robustness and fast response, RAM SMC is proposed. Especially, RAM method is good to apply SMC for WSR control because rotation and movement are timeindependent in RAM control. So each SMC can be designed independently and the controllers are simple and the same type. The MATLAB/SIMULINK simulation is accomplished and the results show that RAM control works well and the robustness around the sliding surface also keeps well. The state also response shows fast and good tracking. The stability of the proposed controllers is proved by the Lyapunov function and we will test under the various disturbances such as ripple, wind, etc. in the future research.

\section{Acknowledgements}

This work was supported by research grants from the Daegu Catholic University in 2017.

\section{References}

[1] Suter, R.B., Rosenberg, O., Loeb, S., Wildman, H. and Long, J.H.J, 1997. Locomotion on the water surface: Propulsive mechanisms of the fisher spider, Journal of Experimental Biology, 200: 2523-2538.

[2] Hu, D.L., Chan, B. and Bush, J.W.M., 2003. The hydrodynamics of water strider locomotion, Nature, 424: 553-666.

[3] Gao, X. and Jiang, L., 2004. Water-repellent legs of water striders, Nature, 432:36. 
[4] Suhr, S.H., Song, Y.S., Lee, S.J. and Sitti, M., 2005. Biologically inspired water strider robot, Robotics: Science and Systems, MIT, Boston.

[5] Bush, J.W.M. and Hu, D.L., 2006. Walking on water: Biolocomotion at the interface, Annual Review of Fluid Mechanics, 38: 339-369.

[6] Song, Y.S., Suhr, S.H. and Sitti, M., 2006. Modeling of the supporting legs for designing biomimetic water strider robots, in Proceedings of IEEE International Conference on Robotics and Automation, Orlando, 2303-2310.

[7] Vella, D. and Metcalfe, P.D., 2007. Surface tension dominated impact, Phycis of Fluids, 19: 072 108-1-11.

[8] Song, Y.S. and Sitti, M., 2007. A highly maneuverable and nontethered water strider robot, in Proceedings of IEEE International Conference on Robotics and Automation, Roma, 980-984.

[9] Chun, K., Kim, J.J., Cho, C.S., Lee, B.L. and Kim, B., 2017. Simple RAM control for water strider robot, submitted at Journal of Engineering and Applied Sciences.

[10] Chun, K., Lee, Y.J. and Choi, B.Y., 1997. A variable structure controller with a PI-type reaching law, Journal of Institute of Control, Robotics and Systems, 3(3): 214-218.

[11] Chun, K. and Sunwoo, M., 2004. Wheel slip control with moving sliding surface for traction control system, International Journal of Automotive Technology, 5(2): 123-133.

[12] Chun, K. and Sunwoo, M., 2005. Wheel slip tracking using moving sliding surface, Proceedings of the Institution of Mechanical Engineers, Part D: Journal of Automobile Engineering, 219(1): 31-41.

[13] Chun, K., Kim, K.T. and Lee, I.S., 2014. Sliding mode yaw contro method for collision avoidance, Journal of Korea Institute of Information Technology, 12(7): 27-34.

[14] Chun, K. and Kim, B., 2015. Robust control with a compensator for inadmissible disturbances, International Journal of Applied Engineering Research, 10(89): 7-11.

[15] Chun, K., Lee, D.S. and Kim, B., 2015. Altitude control with PI SMC for quadcopter, International Journal of Applied Engineering Research, 10(89): 17-21.

[16] Chun, K., Kim, B., Jon, Y.J., Lee, D.I. and Lee, I.S., 2017. Saturation-type reaching law for altitude control, International Journal of Applied Engineering Research, 12(7): 1442-1445. 